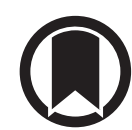

CrossMark

\section{A profile of a retrospective cohort of 22 patients with COVID-19 and active/treated tuberculosis}

To the Editor:

We read with interest the two articles by TADOLINI et al. [1] and STOCHINo et al. [2], which described recent cohorts of either current or former tuberculosis (TB) patients with coronavirus disease 2019 (COVID-19) and studied their clinical course. India has the majority of global burden of $\mathrm{TB}$, along with highest rising number of daily COVID-19 cases in the world $[3,4]$. The information about COVID-19 and active/former TB co-infection reported so far is sparse, but it can be assumed that person with TB, when co-infected with COVID-19, may be at more risk of poor outcomes $[1,5]$. The present study describes the first-ever cohort of current or treated TB patients co-infected with COVID-19 from a high TB burden country, recruited by a tertiary care hospital in India.

This was a retrospective observational study from 1 February 2020 to 14 June 2020, during which a total of 1073 consecutive COVID-19 patients were admitted. Out of these, 22 cases with a diagnosis of active/ treated TB and COVID-19 co-infection were included in the study.

Among 22 patients with COVID-19 and TB co-infection, 13 (59.1\%) patients had active TB (median age (interquartile range (IQR)) 36 (27-59.5) years) and nine (40.9\%) patients had been treated for TB (median age (IQR) 44 (28-51) years) in the past. Among the active TB group, 11 (84.6\%) were females and among the treated TB group, all patients were females. Out of the 13 active TB patients, 12 patients were already receiving anti-TB treatment (ATT) (median duration (IQR) 2 (1-3) months) at the time of admission, while one patient was newly diagnosed with pulmonary TB within a week of admission. The demographic, clinical, radiological and laboratory investigation details, and outcomes of each of the 22 patients, are described in table 1 .

All patients, except one, were symptomatic at the time of presentation. All 12 patients with active TB, who were already receiving ATT at the time of admission, had become almost asymptomatic for TB symptoms. Among them, signs and symptoms attributed to COVID-19 included fever (100\%), dry cough (53.8\%) and dyspnoea (30.8\%) (median (range) duration 2 (2-30) days). Nine treated TB patients were also almost asymptomatic for TB prior to the development of current COVID-19 infection. Among them fever (88.9\%), dry cough (44.4\%) and dyspnoea (33.3\%), respectively were present (median (range) duration of 5 (2-30) days), which could be attributed to COVID-19 disease. Radiological examination, conducted at admission, revealed pulmonary parenchymal fibrosis in all patients in the treated TB group with three (33.3\%) patients having accompanying residual cavitation as well. Among the 13 active TB patients, nine (69.2\%) had pulmonary TB and four (30.8\%) had extra-pulmonary TB. Among the nine active pulmonary TB patients, cavitation was present in three (33.3\%), and six (66.7\%) had parenchymal infiltrates/ consolidation on chest radiography but no cavitation. Among four active extra-pulmonary TB patients, one had cerebral tuberculoma, two had pleural effusion, and one patient had only cervical lymphadenopathy. One active pulmonary TB patient had multidrug-resistant (MDR) TB (isoniazid and rifampicin resistant), receiving conventional MDR treatment regimen as per national guidelines. All treated TB cases had had pulmonary TB.

Lymphopenia was found in only one patient. In all, seven patients (31.8\%) required critical care, 4/13 (30.7\%) in the active TB group and 3/9 (33.3\%) in the treated TB group. All but one patient who required

@ERSpublications

Both tuberculosis and COVID-19 being communicable and prevalent diseases in India, the co-existence can lead to worse outcomes, as seen in this study, where there was high mortality among active as well as treated TB patients with COVID-19 co-infection https://bit.ly/3jHcGbQ

Cite this article as: Gupta N, Ish P, Gupta A, et al. A profile of a retrospective cohort of 22 patients with COVID-19 and active/treated tuberculosis. Eur Respir J 2020; 56: 2003408 [https://doi.org/10.1183/ 13993003.03408-2020]. 


\begin{tabular}{|c|c|c|c|c|c|c|c|c|c|c|c|c|c|}
\hline Patient & $\begin{array}{l}\text { Age } \\
\text { years/ } \\
\text { gender }\end{array}$ & TB status & Site of TB & ATT history & $\begin{array}{l}\text { Clinical } \\
\text { presentation on } \\
\text { admission }\end{array}$ & Comorbidities & $\begin{array}{l}S_{\mathrm{pO2}} \% \text {, } \\
\text { respiratory } \\
\text { rate per } \\
\text { min, GCS, } \\
\quad \text { qSOFA }\end{array}$ & Laboratory findings & $\begin{array}{l}\text { Findings in } \\
\text { chest } \\
\text { radiograph } \\
\text { prior to } \\
\text { Covid-19 }\end{array}$ & $\begin{array}{l}\text { Bilateral new } \\
\text { infiltrates, } \\
\text { consistent with } \\
\text { COVID-19, on CXR }\end{array}$ & $\begin{array}{l}\text { Required } \\
\quad \text { critical } \\
\text { care, } \\
\text { required } \\
\text { IMV }\end{array}$ & $\begin{array}{l}\text { Duration of } \\
\text { hospital } \\
\text { stay } \\
\text { days }\end{array}$ & $\begin{array}{l}\text { Endpoint } \\
\quad \text { attained } \\
\text { (discharged/ } \\
\text { died) }\end{array}$ \\
\hline 1 & 54/Male & Treated & РTB & $\begin{array}{l}\text { Completed DS-TB } \\
\text { regimen } 2 \text { years } \\
\text { earlier }\end{array}$ & Fever for 2 days & $\begin{array}{l}\text { Hypertension, } \\
\text { hypothyroidism }\end{array}$ & $\begin{array}{l}\mathrm{S}_{\mathrm{PO}_{2}} 98, \\
\mathrm{RR} 19 \\
\text { GCS } 15 \\
\text { qSOFA } 0\end{array}$ & $\begin{array}{l}\text { TLC } 4800 \text { (488\% } \\
\text { (ymphocytes) }\end{array}$ & $\begin{array}{l}\text { Right lung } \\
\text { fibrosis }\end{array}$ & Yes & No & 15 & Discharged \\
\hline 2 & 58/Male & Treated & PTB & $\begin{array}{l}\text { Completed DS-TB } \\
\text { regimen } 1 \text { year } \\
\text { earlier }\end{array}$ & Fever for 5 days & & $\begin{array}{l}\mathrm{S}_{\mathrm{Po}_{2}} 25, \\
\quad \mathrm{RR} 24 \\
\text { GCS } 15 \\
\text { qSOFA } 1\end{array}$ & $\begin{array}{l}\text { TLC } 8500 \text { (40\% } \\
\text { (ymphocytes) }\end{array}$ & $\begin{array}{l}\text { Right lung } \\
\text { fibrosis }\end{array}$ & Yes & No & 7 & Discharged \\
\hline 3 & 44/Male & Treated & РTB & $\begin{array}{l}\text { Completed DS-TB } \\
\text { regimen } 2 \text { years } \\
\text { earlier }\end{array}$ & Fever for 6 days & & $\begin{array}{l}\mathrm{SOO}_{2} 98, \\
\mathrm{RR}_{2} 20, \\
\text { GCS 15, } \\
\text { qSOFA } 0\end{array}$ & $\begin{array}{l}\text { TLC } 4100 \text { (31\% } \\
\text { (ymphocytes) }\end{array}$ & Left lung fibrosis & No & No & 15 & Discharged \\
\hline 4 & 19/Male & Treated & PTB & $\begin{array}{l}\text { Completed DS-TB } \\
\text { regimen } 1 \text { year } \\
\text { earlier }\end{array}$ & Asymptomatic & & $\begin{array}{l}\mathrm{S}_{\mathrm{PO}_{2}} 95 \\
\quad \mathrm{RR} 18 \\
\text { GCS } 15 \\
\text { qSOFA }\end{array}$ & $\begin{array}{l}\text { TLC } 4400 \text { ( } 45 \% \\
\text { (ymphocytes) }\end{array}$ & Left lung fibrosis & No & No & 15 & Discharged \\
\hline 5 & 26/Male & Treated & PTB & $\begin{array}{l}\text { Completed DS-TB } \\
\text { regimen } 2 \text { years } \\
\text { earlier }\end{array}$ & $\begin{array}{l}\text { Fever and cough } \\
3 \text { days }\end{array}$ & & $\begin{array}{l}\mathrm{S}_{\mathrm{PO}_{2}} 95, \\
\quad \mathrm{RR} 18 \\
\text { GCS } 15 \\
\text { qSOFA } 0\end{array}$ & $\begin{array}{l}\text { TLC } 5300 \text { (30\% } \\
\text { (ymphocytes) }\end{array}$ & $\begin{array}{l}\text { Right lung } \\
\text { fibrosis }\end{array}$ & No & No & 5 & Discharged \\
\hline 6 & 48/Male & Treated & PTB & $\begin{array}{l}\text { Completed DS-TB } \\
\text { regimen } 1 \text { year } \\
\text { earlier }\end{array}$ & $\begin{array}{l}\text { Breathlessness for } \\
2 \text { days, fever and } \\
\text { cough for } \\
2 \text { weeks }\end{array}$ & Diabetes mellitus & $\begin{array}{l}\mathrm{S}_{\mathrm{PO}_{2}} 80 \\
\quad \mathrm{RR} 38 \\
\text { GCS 4, } \\
\text { qSOFA } 3\end{array}$ & $\begin{array}{l}\text { TLC } 8200 \text { (42\% } \\
\text { (ymphocytes) }\end{array}$ & $\begin{array}{l}\text { Left lung fibrosis } \\
\text { and left } \\
\text { upper zone } \\
\text { cavity }\end{array}$ & Yes & $\begin{array}{l}\text { Required } \\
\text { critical } \\
\text { care and } \\
\text { IMV }\end{array}$ & $<1$ & Died \\
\hline 7 & 30/Male & Treated & РTB & $\begin{array}{l}\text { Completed DS-TB } \\
\text { regimen } 2 \text { months } \\
\text { earlier }\end{array}$ & Fever for 3 days & & $\begin{array}{l}\mathrm{S}_{\mathrm{PO}_{2}} 99 \\
\quad \mathrm{RR} 20 \\
\text { GCS 15, } \\
\text { qSOFA } 0\end{array}$ & $\begin{array}{l}\text { TLC } 7700 \text { (42\% } \\
\text { (ymphocytes) }\end{array}$ & $\begin{array}{l}\text { Right lung } \\
\text { fibrosis and } \\
\text { right upper } \\
\text { zone } \\
\text { cavitation }\end{array}$ & No & No & 16 & Discharged \\
\hline 8 & 38/Male & Treated & PTB & $\begin{array}{l}\text { Completed DS-TB } \\
\text { regimen } 6 \text { years } \\
\text { earlier }\end{array}$ & $\begin{array}{l}\text { Fever, cough and } \\
\text { breathlessness } \\
\text { for } 5 \text { days }\end{array}$ & & $\begin{array}{l}\mathrm{S}_{\mathrm{PO}_{2}} 81 \\
\text { RR } 38 \\
\text { GCS 3, } \\
\text { qSOFA } 3\end{array}$ & & Left lung fibrosis & Yes & $\begin{array}{l}\text { Required } \\
\text { critical } \\
\text { care and } \\
\text { IMV }\end{array}$ & $<1$ & Died \\
\hline 9 & 45/Male & Treated & PTB & $\begin{array}{l}\text { Completed DS-TB } \\
\text { regimen } 6 \text { months } \\
\text { earlier }\end{array}$ & $\begin{array}{l}\text { Breathlessness for } \\
2 \text { days, fever for } \\
5 \text { days, cough for } \\
1 \text { month }\end{array}$ & & $\begin{array}{l}\mathrm{SOO}_{\mathrm{PO}_{2}} 78, \\
\quad \mathrm{RR} 32, \\
\text { GCS } 3 \\
\text { qSOFA } 3\end{array}$ & $\begin{array}{l}\text { ABGA, respiratory } \\
\text { acidosis }\end{array}$ & $\begin{array}{l}\text { Left lung fibrosis } \\
\text { and upper } \\
\text { and mid zone } \\
\text { cavitation }\end{array}$ & Yes & $\begin{array}{l}\text { Required } \\
\text { critical } \\
\text { care and } \\
\text { IMV }\end{array}$ & $<1$ & Died \\
\hline 10 & 45/Male & Active & PTB & $\begin{array}{l}\text { On DS-TB regimen for } \\
1 \text { month }\end{array}$ & $\begin{array}{l}\text { Fever, headache, } \\
\text { cough, } \\
\text { breathlessness } \\
\text { for } 3 \text { days }\end{array}$ & & $\begin{array}{l}\mathrm{S}_{\mathrm{pO}_{2}} 86, \\
\quad \mathrm{RR} 32, \\
\text { GCS 3, } \\
\text { qSOFA } 2\end{array}$ & $\begin{array}{l}\text { Hb } 10.4, \text { TLC } 15000 \\
\text { (10\% lymphocytes), } \\
\text { Plt } 180000 \text {, blood } \\
\text { urea } 125 \text {, creatinine } \\
\text { 6.5, total bilirubin } \\
\text { 0.5, AST 456, ALT } \\
178\end{array}$ & $\begin{array}{l}\text { Left upper zone } \\
\text { cavitation }\end{array}$ & Yes & $\begin{array}{l}\text { Required } \\
\text { critical } \\
\text { care and } \\
\text { IMV }\end{array}$ & 2 & Died \\
\hline 11 & 26/Male & Active & PTB & $\begin{array}{l}\text { On DS-TB regimen for } \\
3 \text { months }\end{array}$ & Fever for 2 days & & $\begin{array}{l}\mathrm{S}_{\mathrm{PO}_{2}} 94, \\
\mathrm{RR} 20 \\
\text { GCS } 15 \\
\text { qSOFA }\end{array}$ & $\begin{array}{l}\text { TLC } 8000 \text { (20\% } \\
\text { (ymphocytes) }\end{array}$ & $\begin{array}{l}\text { Right upper zone } \\
\text { infiltrates }\end{array}$ & No & No & 20 & Discharged \\
\hline 12 & 63/Male & Active & PTB & $\begin{array}{l}\text { On DS-TB regimen for } \\
2 \text { months }\end{array}$ & $\begin{array}{l}\text { Breathlessness for } \\
5 \text { days, fever for } \\
12 \text { days }\end{array}$ & & $\begin{array}{l}\mathrm{S}_{\mathrm{PO}_{2}} 92, \\
\mathrm{RR} 24 \\
\text { GCS } 15 \\
\text { qSOFA } 1\end{array}$ & $\begin{array}{l}\text { TLC } 9000 \text { (40\% } \\
\text { (ymphocytes) }\end{array}$ & $\begin{array}{l}\text { Right upper zone } \\
\text { cavitation }\end{array}$ & Yes & No & 24 & Discharged \\
\hline
\end{tabular}




\begin{tabular}{|c|c|c|c|c|c|c|c|c|c|c|c|c|c|}
\hline Patient & $\begin{array}{l}\text { Age } \\
\text { years/ } \\
\text { gender }\end{array}$ & TB status & Site of TB & ATT history & $\begin{array}{l}\text { Clinical } \\
\text { presentation on } \\
\text { admission }\end{array}$ & Comorbidities & $\begin{array}{l}S_{\mathrm{PO} 2} \% \text {, } \\
\text { respiratory } \\
\text { rate per } \\
\text { min, GCS, } \\
\text { qSOFA }\end{array}$ & Laboratory findings & $\begin{array}{l}\text { Findings in } \\
\text { chest } \\
\text { radiograph } \\
\text { prior to } \\
\text { COVID-19 }\end{array}$ & $\begin{array}{l}\text { Bilateral new } \\
\text { infiltrates, } \\
\text { consistent with } \\
\text { coVID-19, on CXR }\end{array}$ & $\begin{array}{l}\text { Required } \\
\quad \text { critical } \\
\text { care, } \\
\text { required } \\
\text { IMV }\end{array}$ & $\begin{array}{l}\text { Duration of } \\
\text { hospital } \\
\text { stay } \\
\text { days }\end{array}$ & $\begin{array}{l}\text { Endpoint } \\
\quad \text { attained } \\
\text { (discharged/ } \\
\text { died) }\end{array}$ \\
\hline 13 & 29/Male & Active & PTB & $\begin{array}{l}\text { On DS-TB regimen for } \\
1 \text { month }\end{array}$ & Fever for 2 days & & $\begin{array}{l}\mathrm{S}_{\mathrm{PO}_{2}} 98, \\
\mathrm{RR} 16 \\
\text { GCS 15, } \\
\text { qSOFA O }\end{array}$ & $\begin{array}{l}\text { TLC } 8500(30 \% \\
\text { lymphocytes) }\end{array}$ & $\begin{array}{l}\text { Left mid zone } \\
\text { consolidation }\end{array}$ & No & No & 15 & Discharged \\
\hline 14 & 65/Female & Active & РТВ & $\begin{array}{l}\text { On MDR-TB regimen for } \\
1 \text { year }\end{array}$ & $\begin{array}{l}\text { Fever, cough and } \\
\text { breathlessness } \\
\text { for } 5 \text { days }\end{array}$ & $\begin{array}{l}\text { Diabetes mellitus, } \\
\text { hypertension }\end{array}$ & $\begin{array}{l}\mathrm{S}_{\mathrm{PO}_{2}} 76 \\
\quad \mathrm{RR} 34 \\
\text { GCS 4, } \\
\text { qSOFA } 3\end{array}$ & $\begin{array}{l}\text { ABGA, high anion gap } \\
\text { metabolic acidosis, } \\
\text { diabetic ketoacidosis } \\
\text { with blood sugar } \\
\text { value of } \\
470 \mathrm{mg}^{-1 L^{-1}} \text {, blood } \\
\text { urea } 70, \text { creatinine } \\
3.8, \text { TLC } 17300 \text { (22\% } \\
\text { lymphocytes) }\end{array}$ & $\begin{array}{l}\text { Right upper, mid } \\
\text { and lower } \\
\text { zone } \\
\text { consolidation }\end{array}$ & Yes & $\begin{array}{l}\text { Required } \\
\text { critical } \\
\text { care and } \\
\text { IMV }\end{array}$ & 1 & Died \\
\hline 15 & 29/Male & Active & EPTB (CNS-TB) & $\begin{array}{l}\text { On ATT for CNS-TB for } \\
12 \text { months }\end{array}$ & $\begin{array}{l}\text { Fever and cough for } \\
1 \text { month with } \\
\text { weight loss }\end{array}$ & Seizure disorder & $\begin{array}{l}\mathrm{S}_{\mathrm{PO}_{2}} 90 \\
\mathrm{RR} 24 \\
\text { GCS 15, } \\
\text { qSOFA } 1\end{array}$ & $\begin{array}{l}\text { TLC } 800 \text { (26\% } \\
\text { lymphocytes) }\end{array}$ & & Yes & $\begin{array}{l}\text { Required } \\
\text { critical } \\
\text { care but } \\
\text { not IMV" }\end{array}$ & 20 & Discharged \\
\hline 16 & 26/Male & Active & PTB & $\begin{array}{l}\text { On DS-TB regimen for } \\
4 \text { months }\end{array}$ & $\begin{array}{l}\text { Fever and cough for } \\
2 \text { days }\end{array}$ & & $\begin{array}{l}\mathrm{S}_{\mathrm{PO}_{2}} 95, \\
\mathrm{RR} 20 \\
\text { GCS } 15 \\
\text { qSOFA }\end{array}$ & $\begin{array}{l}\text { TLC } 8100 \text { (40\% } \\
\text { (ymphocytes) }\end{array}$ & $\begin{array}{l}\text { Right upper zone } \\
\text { infiltrates }\end{array}$ & Yes & No & 8 & Discharged \\
\hline 17 & 56/Male & Active & PTB & $\begin{array}{l}\text { On DS-TB regimen for } \\
3 \text { months }\end{array}$ & $\begin{array}{l}\text { Fever and cough for } \\
3 \text { days }\end{array}$ & Hypertension & $\begin{array}{l}\mathrm{S}_{\mathrm{PO}_{2}} 93, \\
\mathrm{RR} 18 \\
\text { GCS } 15 \\
\text { qSOFA }\end{array}$ & $\begin{array}{l}\text { TLC- } 9700 \text { (36\% } \\
\text { Lymphocytes) }\end{array}$ & $\begin{array}{l}\text { Left upper zone } \\
\text { infiltrates }\end{array}$ & Yes & No & 7 & Discharged \\
\hline 18 & 36/Male & Active & $\begin{array}{l}\text { Disseminated TB } \\
\text { (pulmonary } \\
\text { and } \\
\text { abdominal) }\end{array}$ & $\begin{array}{l}\text { On DS-TB regimen for } \\
2 \text { months }\end{array}$ & Fever for 2 days & & $\begin{array}{l}\mathrm{S}_{\mathrm{PO}_{2}} 98, \\
\mathrm{RR} 18 \\
\text { GCS } 15 \\
\text { qSOFA }\end{array}$ & $\begin{array}{l}\text { TLC } 7900 \text { (47\% } \\
\text { lymphocytes) }\end{array}$ & $\begin{array}{l}\text { Left upper zone } \\
\text { cavitation }\end{array}$ & Yes & No & 10 & Discharged \\
\hline 19 & 40/Male & $\begin{array}{l}\text { Active and newly } \\
\text { diagnosed } \\
\text { concommitantly } \\
\text { with COVID-19 }\end{array}$ & $\begin{array}{l}\text { EPTB (pleural } \\
\text { effusion) }\end{array}$ & $\begin{array}{l}\text { Initiated on DS-TB } \\
\text { regimen }\end{array}$ & $\begin{array}{l}\text { Fever and cough for } \\
2 \text { days }\end{array}$ & & $\begin{array}{l}\mathrm{S}_{\mathrm{PO}_{2}} 99, \\
\mathrm{RR} 16, \\
\text { GCS 15, } \\
\text { qSOFA } 0\end{array}$ & $\begin{array}{l}\text { TLC } 8300(39 \% \\
\text { lymphocytes) }\end{array}$ & $\begin{array}{l}\text { Left pleural } \\
\text { effusion }\end{array}$ & No & No & 10 & Discharged \\
\hline 20 & 21/Male & Active & $\begin{array}{l}\text { EPTB Icervical } \\
\text { LN-TB) }\end{array}$ & $\begin{array}{l}\text { On DS-TB regimen for } \\
1 \text { month }\end{array}$ & Fever for 2 days & & $\begin{array}{l}\mathrm{S}_{\mathrm{pO}_{2}} 75, \\
\quad \mathrm{RR} 36, \\
\text { GCS } 4 \\
\text { qSOFA } 1\end{array}$ & $\begin{array}{l}\text { ABGA, high anion gap } \\
\text { metabolic acidosis, } \\
\text { blood urea } 72, \\
\text { creatinine } 3.8, \mathrm{TLC} \\
19600 \text { (27\% } \\
\text { lymphocytes) }\end{array}$ & & Yes & $\begin{array}{l}\text { Required } \\
\text { critical } \\
\text { care and } \\
\text { IMV }\end{array}$ & 5 & Died \\
\hline 21 & 28/Female & Active & РТВ & $\begin{array}{l}\text { On DS-TB regimen for } \\
1 \text { month }\end{array}$ & Fever for 2 days & & $\begin{array}{l}\mathrm{S}_{\mathrm{PO}_{2}} 95, \\
\mathrm{RR} 18 \\
\text { GCS 15, } \\
\text { qSOFA O }\end{array}$ & $\begin{array}{l}\text { TLC } 6300 \text { (32\% } \\
\text { (ymphocytes) }\end{array}$ & $\begin{array}{l}\text { Left upper zone } \\
\text { infiltrates }\end{array}$ & No & No & 12 & Discharged \\
\hline 22 & 67/Male & Active & $\begin{array}{l}\text { EPTB (pleural } \\
\text { effusion) }\end{array}$ & $\begin{array}{l}\text { On DS-TB regimen for } \\
2 \text { months }\end{array}$ & $\begin{array}{l}\text { Fever, cough and } \\
\text { breathlessness } \\
\text { for } 3 \text { days }\end{array}$ & $\begin{array}{l}\text { Diabetes mellitus, } \\
\text { hypertension, } \\
\text { seizure } \\
\text { disorder }\end{array}$ & $\begin{array}{l}\mathrm{S}_{\mathrm{PO}_{2}} 95, \\
\mathrm{RR} 20 \\
\text { GCS 15, } \\
\text { qSOFA O }\end{array}$ & $\begin{array}{l}\text { TLC } 3700 \text { (34\% } \\
\text { lymphocytes) }\end{array}$ & $\begin{array}{l}\text { Right pleural } \\
\text { effusion }\end{array}$ & Yes & No & 14 & Discharged \\
\hline
\end{tabular}

PTB: pulmonary tuberculosis; EPTB: extra-pulmonary tuberculosis; CNS-TB: central nervous system tuberculosis; LN-TB: lymph node tuberculosis; ATT: anti-tubercular treatment; DS-TB: drug sensitive tuberculosis; MDR-TB: multi-drug resistant tuberculosis; $\mathrm{S}_{\mathrm{pO}_{2}}$ : oxygen saturation by pulse oximetry; GCS: Glasgow coma scale score; qSOFA: quick sepsis related organ failure score; TLC: total leukocyte count lunit: per $\mathrm{mm}^{3}$ ): ABGA: arterial blood gas analysis; Hb: haemoglobin (unit: $\mathrm{g} \cdot \mathrm{dL}^{-1}$ ): $\mathrm{Plt}$ : platelet count (unit: per $\mathrm{mm}^{3}$ ), $\mathrm{AST}$ : aspartate transaminase (unit: IU. $L^{-1}$ ); ALT: alanine transaminase (unit: IU. $L^{-1}$ ); CXR: chest radiograph; IMV: invasive mechanical ventilation. Laboratory parameters for blood urea, creatinine and bilirubin are provided in units of $\mathrm{mg} \cdot \mathrm{dL}^{-1}$. \# : patient's duration of stay in intensive care unit was 5 days, and on ward was 15 days. 
critical care also required invasive mechanical ventilation. Among these, $3 / 13$ (23.1\%) patients were from the active TB group and 3/9 (33.3) patients were from the treated TB group. All these six patients died; this group also included one MDR-TB patient.

All six patients who died had hypoxaemia and a Glasgow Coma Scale (GCS) score of 3-4 on admission. Quick sepsis-related organ failure scores were 3 in four patients, and 2 and 1 in one patient each. Death in all deceased patients was attributed to COVID-19 co-infection, as all were otherwise responding clinically and radiologically to ATT in the active TB group or were clinically stable in the treated TB group. Comorbid diabetes mellitus was observed in $3 / 22$ (13.6\%) patients and two (66.7\%) among them died. None of the patients had HIV. Of the 22 patients, 16 patients $(72.7 \%)$ were discharged. During the study period, 14 days of admission was mandatory for COVID-19 patients as per national guidelines [6]. Among those discharged, the mean \pm SD duration of stay was $13.3 \pm 5.3$ days.

In the present series, among 22 patients with TB and COVID-19 co-infection, the overall mortality rate was $27.3 \%$. This mortality rate, though preliminary, is higher as compared to other studies by TADOLINI et al. [1] (12.3\%) and MоттA et al. [7] (11.6\%) in TB and COVID-19 co-infected patients. A review by ONG et al. [8] also found a higher mortality in TB with COVID-19. In India, a mortality rate of around $2.3 \%$ has been observed among COVID-19 patients, including patients with comorbid conditions such as diabetes, hypertension, malignancy and tuberculosis, etc. [4]. This higher mortality in TB and COVID-19 co-infection could be explained by damage to the lungs by fibrosis or cavitation in treated TB cases, or by active TB disease with superimposed insult of COVID-19 co-infection leading to further deterioration of already compromised lung function.

In the initial cohort of 40 COVID-19 patients who had been admitted to the authors' centre up to 31 March 2020, no patient had active or previously treated TB; however, over the subsequent 6 weeks, the incidence of active and treated TB went up to 1.21 and 0.83 per 100 hospital admissions for COVID-19, respectively [9].

The limitations of the study were that the role of pathological and biochemical factors, such as D-dimer, C reactive protein, IL-6 and ferritin, etc., and use of investigational drugs, such as tocilizumab, remdesivir, favipiravir and steroids, for patient management were not studied, as neither of these were a component of national treatment guidelines during the study period [6]. Also, because of the small sample size, analysis of various risk factors was not carried out.

In conclusion, patients with treated or active TB may be considered another vulnerable group for COVID-19 and may require special attention and appropriate preventive measures for development of COVID-19. Further, a high mortality, along with a greater need for critical care, was found in active as well as treated TB patients co-infected with COVID-19.

Nitesh Gupta ${ }^{1}$, Pranav Ish ${ }^{1}$, Amitesh Gupta ${ }^{2}$, Nipun Malhotra ${ }^{1}$, Jose Antonio Caminero ${ }^{3,4}$, Rupak Singla ${ }^{5}$, Rohit Kumar ${ }^{1}$, Siddharth Raj Yadav ${ }^{1}$, Nishanth Dev $^{6}$, Sumita Agrawal, Santvana Kohli $\oplus^{8}$, M.K. Sen ${ }^{1}$, Shibdas Chakrabarti ${ }^{1}$ and N.K. Gupta ${ }^{1}$

${ }^{1}$ Pulmonary Medicine, Safdarjung Hospital, New Delhi, India. ${ }^{2}$ National Institute of Tuberculosis and Respiratory Diseases, New Delhi, India. ${ }^{3}$ Dept of Pneumology, Dr Negrín University Hospital of Gran Canaria, Las Palmas de Gran Canaria, Spain. ${ }^{4}$ MDR-TB Unit, Tuberculosis Division, International Union against Tuberculosis and Lung Disease, Paris, France. ${ }^{5}$ Dept of Tuberculosis and Respiratory Diseases, National Institute of Tuberculosis and Respiratory Diseases, New Delhi, India. ${ }^{6}$ Medicine, Safdarjung Hospital, New Delhi, India. ${ }^{7}$ Pulmonary Medicine, Medipulse Hospital, Jodhpur, India. ${ }^{8}$ Anaesthesiology, Safdarjung Hospital, New Delhi, India.

Correspondence: Nitesh Gupta, Room number 638, Dept of Pulmonary Medicine, Safdarjung Hospital, New Delhi110029, India. E-mail: niteshgupta2107@gmail.com

Received: 6 Sept 2020 | Accepted after revision: 13 Oct 2020

Author contributions: All the 14 authors made substantial contributions to the conception or design of the work; or the acquisition, analysis, or interpretation of data for the work; or drafting the work or revising it critically for important intellectual content; and towards final approval of the version to be published; and agree to be accountable for all aspects of the work in ensuring that questions related to the accuracy or integrity of any part of the work are appropriately investigated and resolved.

Declaration of patient consent: the authors certify that they have obtained written informed consent from the patients to publish their personal details and follow up. The patients understand that their name and initials will not be published but, anonymity cannot be guaranteed. Ethics committee approval was not required as it was a retrospective observational study. 


\section{References}

1 Tadolini M, Codecasa LR, García-García J-M, et al. Active tuberculosis, sequelae and COVID-19 co-infection: first cohort of 49 cases. Eur Respir J 2020; 56: 2001398.

2 Stochino C, Villa S, Zucchi P, et al. Clinical characteristics of COVID-19 and active tuberculosis co-infection in an Italian reference hospital. Eur Respir J 2020; 56: 2001708.

3 Ministry of Health and Family Welfare. www.mohfw.gov.in/ Date last accessed: 10 August 2020.

4 Central TB Division: Ministry of Health and Family Welfare. India TB Report. New Delhi, Ministry of Health and Family Welfare, 2020. Available from https://tbcindia.gov.in/WriteReadData/1892s/India\%20TB\%20Report\%202020. pdf Date last accessed: 10 August 2020.

5 Liu Y, Bi L, Chen Y, et al. Active or latent tuberculosis increases susceptibility to COVID-19 and disease severity. medRxiv 2020; preprint [https://doi.org/10.1101/2020.03.10.20033795].

6 Government of India, Ministry of Health and Family Welfare, Directorate General of Health Services. Revised National Clinical Management Guideline for COVID-19. www.mohfw.gov.in/pdf/RevisedNationalClinical ManagementGuidelineforCOVID1931032020.pdf. Date last accessed: 10 August 2020. Date last updated: 31 March 2020.

7 Motta I, Centis R, D’Ambrosio L, et al. Tuberculosis, COVID-19 and migrants: preliminary analysis of deaths occurring in 69 patients from two cohorts. Pulmonology 2020; 26: 233-240.

8 Ong CWM, Migliori GB, Raviglione M, et al. Epidemic and pandemic viral infections: impact on tuberculosis and the lung. A consensus by the World Association for Infectious Diseases and Immunological Disorders (WAidid), Global Tuberculosis Network (GTN) and members of ESCMID Study Group for Mycobacterial Infections (ESGMYC). Eur Respir J 2020; 56: 2001727.

9 Gupta N, Agrawal S, Ish P, et al. Clinical and epidemiologic profile of the initial COVID-19 patients at a tertiary care centre in India. Monaldi Arch Chest Dis 2020; 90: 1294. 